\title{
Photoreceptor Outer Segment on Internal Limiting Membrane after Macular Hole Surgery: Implications for Pathogenesis
}

\author{
Michael E. Grinton Maria T. Sandinha David H.W. Steel \\ Sunderland Eye Infirmary, Sunderland, UK
}

\section{Key Words}

Macular hole · Tangential vitreomacular traction · Internal limiting membrane .

Photoreceptors

\begin{abstract}
Purpose: This report presents a case, which highlights key principles in the pathophysiology of macular holes. It has been hypothesized that anteroposterior (AP) and tangential vitreous traction on the fovea are the primary underlying factors causing macular holes [Nischal and Pearson; in Kanski and Bowling: Clinical Ophthalmology: A Systemic Approach, 2011, pp 629631]. Spectral domain optical coherence tomography (OCT) has subsequently corroborated this theory in part but shown that AP vitreofoveal traction is the more common scenario [Steel and Lotery: Eye 2013;27:1-21]. Methods: This study was conducted as a single case report. Results: A 63-year old female presented to her optician with blurred and distorted vision in her left eye. OCT showed a macular hole with a minimum linear diameter of $370 \mu \mathrm{m}$, with persistent broad vitreofoveal attachment on both sides of the hole edges. The patient underwent combined left phacoemulsification and pars plana vitrectomy, internal limiting membrane (ILM) peel and gas injection. The ILM was examined by electron microscopy and showed the presence of a cone outer segment on the retinal side. Post-operative OCT at 11 weeks showed a closed hole with recovery of the foveal contour and good vision. Conclusion: Our case shows the presence of a photoreceptor outer segment on the retinal side of the ILM and reinforces the importance of tangential traction in the development of some macula holes. The case highlights the theory of transmission of inner retinal forces to the photoreceptors via Müller cells and how a full thickness macular hole defect can occur in the absence of AP vitreomacular traction.

(C) 2015 S. Karger AG, Basel
\end{abstract}

KARGER 125/s $\begin{aligned} & \text { Michael Grinton, MBChB } \\ & \text { Sunderland Eye Infirmary } \\ & \text { Queen Alexandra Road } \\ & \text { Sunderland, Tyne and Wear SR2 9HP (UK) } \\ & \text { E-Mail Michael.Grinton @ gmail.com }\end{aligned}$


Grinton et al.: Photoreceptor Outer Segment on Internal Limiting Membrane after Macular Hole Surgery: Implications for Pathogenesis

\section{Background}

Johnson and Gass [1] hypothesized that macular holes were caused by shrinkage of adherent cortical vitreous at the fovea with tangential traction causing a circumscribed foveolar detachment with later dehiscence of the foveola retina at the umbo to cause a hole. Their classification system based on fundus biomicroscopy is still in common use. Subsequent optical coherence tomography (OCT) studies, however, have suggested that anteroposterior (AP) traction with focal vitreomacular traction and localized inner and outer retinal dehiscence is important in the initiation of macular holes [2].

We report a macular hole case with complete perifoveal vitreous attachment without any separation around the macular hole. Post-operative internal limiting membrane (ILM) analysis revealed a photoreceptor outer segment on the retinal side of the ILM. Our case further substantiates the theory of outer retinal movement to the inner retinal surface during macular hole formation. Furthermore we show that this can occur in the absence of overt AP retinal traction. Although there was loss of neuroretinal tissue, there was a successful outcome with surgery, contrary to what had been suggested in some studies [3].

\section{Case Presentation}

A 63-year old female with no past ophthalmic history presented with a 4-month history of distortion and blurred vision in her left eye. Spectral OCT (SD OCT) showed a left stage 3 macular hole (fig. 1) with a minimum linear diameter of $370 \mu \mathrm{m}$. Snellen visual acuity (VA) was $-6 / 6$ N5 in the right eye and $-6 / 36$ N12 in the left eye.

\section{Treatment}

The patient underwent combined phacoemulsification with lens implant, 25-gauge pars plana vitrectomy with ILM peel and gas injection (sulfur hexafluoride 25\%) at the Sunderland Eye Infirmary, UK. The ILM was stained with brilliant blue G (ILM Blue; DORC International, Zuidland, The Netherlands) and peeled atraumatically using forceps and a pinch peeling technique (Grieshaber DSP 25-gram end gripping forceps; Alcon). During the operation, the ILM was carefully removed from the eye, immediately fixed in $2 \%$ glutaraldehyde in 0.1 $\mathrm{M}$ sodium cacodylate buffer and sent for transmission electron microscopy (EM). The patient postured face down for $5 \mathrm{~h}$ for 5 days. The patient consented for the ILM sample to be examined by EM

\section{Outcome and Follow Up}

Post-operative OCT at 2 weeks showed a closed hole (fig. 1) and VAs were recorded as $6 / 6$ in the right eye and $-6 / 19$ in the left eye.

The patient was reviewed in the clinic again 11 weeks after the operation, and OCT was performed showing reformation of the foveal contour and closure of the hole. VA in the left eye had improved to 6/9-1 with the patient describing an improvement in her vision. A photoreceptor outer segment with the outer segment discs is clearly seen on a transmission EM picture of the ILM (fig. 2). 
Grinton et al.: Photoreceptor Outer Segment on Internal Limiting Membrane after Macular Hole Surgery: Implications for Pathogenesis

\section{Discussion}

Patients with macular holes often present with impairment of central vision. Macular holes are more common in women and have a peak incidence of onset in the 7th decade [4]. There is a high success rate ( $>90 \%$ [5]) for surgical closure of macular holes, and the present case shows resolution of the hole after vitrectomy surgery.

In 1988, Johnson and Gass [1] hypothesized that macular holes were caused by shrinkage of the adherent vitreous at the fovea causing a circumscribed foveolar detachment and dehiscence of the foveola retina at the umbo. The authors hypothesized that pre-retinal opacities, often seen on biomicroscopy, known as 'opercula', were the result of the foveolar detachment and were composed of neuroretinal tissue.

SD OCT studies have subsequently corroborated this theory in part but shown that AP vitreofoveal traction with perifoveal PVD is the more common scenario [6]. It is thought that these inner retinal forces are transmitted to the outer retina with elevation of the cone outer segment tips (COST) towards the inner retinal surface [7]. Indeed, further studies of avulsed opercula attached to the posterior hyaloid face from the foveolar area and retrieved at the time of surgery have shown the presence of photoreceptor components [3].

The main finding in this case is the presence of a cone outer segment on the retinal side of the ILM. Although Ezra et al. [3] showed the presence of neurites and synaptic complexes typical of cone photoreceptors on EM in the opercula they studied, to our knowledge, no studies have shown the presence of photoreceptor outer segments in an operculum or on the ILM. The presence of an outer segment on the ILM and neurites in opercula can provide clues to the pathogenesis of macular holes. Although there has been a substantial amount of research on the vitreomacular interface and its subsequent role in the pathophysiology of macular holes, the exact relative role of perifoveal vitreous detachment and tangential traction is still not fully elucidated. Takahashi et al. [6] studied the vitreoretinal interface in patients with macular holes without a complete PVD (i.e. Gass stage 3 or less) on SD OCT. They observed focal vitreomacular attachment (VMA), or an avulsed operculum suggestive of previous AP traction, in 59 of 64 eyes. Theodossiadis et al. [8] used a mathematical approach to explain the outer retinal effects of focal AP vitreomacular traction. They described that in narrow vitreofoveal attachment, forces will be most readily transmitted to the outer retina as compared to those with broader adhesion. Indeed it has been observed that in some patients with early macular hole formation and VMA the COST line is elevated in the centre of the fovea and appears to merge with the inner/outer segment junction [9]. As the hole forms, the elevated COST area can become avulsed from the outer retina and adherent to an attached operculum as a triangular shape on OCT. Another common observation as the hole forms further is extension of the ellipsoid zone and external limiting membrane anteriorly up the sides of the macular hole edges (fig. 3). The findings of Takahashi et al. [6] therefore mean that the presence of photoreceptor material in surgically retrieved opercula could be explained by the presence of anteroposterior vitreoretinal traction in the majority of patients with macular holes.

In the case presented herein, however, the patient did not have focal VMA, and SD OCT showed a zone of at least $1,000 \mu \mathrm{m}$ all around the circumference of the fovea where the vitreous was attached to the retina. Therefore, this case suggests that the same mechanism of outer retinal traction can occur in the absence of focal AP traction. We hypothesize that tangential traction with perifoveal vitreous adhesion led to outer retinal traction via the retinal spanning foveal Müller cells and movement of the cone outer segment observed from the outer retina to a point on the edge of the hole deep to the ILM. 
Grinton et al.: Photoreceptor Outer Segment on Internal Limiting Membrane after Macular Hole Surgery: Implications for Pathogenesis

Although this is the mechanism that we have hypothesized to explain our findings, other alternatives are possible. One explanation would be that surgical manipulation at the vitreomacular interface while peeling the ILM caused the ILM to pick up a photoreceptor outer segment and separate it from the other outer retinal components. Another explanation of the mechanism of our findings is that there still could be significant anteroposterior traction at the vitreomacular interface despite there being more than 1,000 $\mu \mathrm{m}$ of attached vitreous at either side of the fovea. The broad attachment could therefore still cause anterior movement of the outer retinal components along with or instead of the tangential traction that we have hypothesized.

Other studies have reported that the outcome of surgery correlates with the presence of outer retinal material in the opercula. Although there was clearly a disruption of the integrity of the photoreceptors during the formation of the hole, the patient still had an excellent visual outcome.

\section{Statement of Ethics}

No funding was received for this work. Informed consent was obtained from the patient for publication of this case report and accompanying images.

\section{Disclosure Statement}

The authors have no conflicts of interest and no proprietary interest.

\section{References}

1 Johnson R, Gass J: Idiopathic macular holes. Observations, stages of formation, and implications for surgical intervention. Ophthalmology 1988;95:917-924.

-2 Steel D, Lotery A: Idiopathic vitreomacular traction and macular hole: a comprehensive review of pathophysiology, diagnosis, and treatment. Eye 2013;27:1-21.

-3 Ezra E, Munro P, Charteris D, et al: Macular hole opercula. Ultrastructural features and clinicopathological correlation. Arch Ophthalmol 1997;115:1381-1387.

4 Nischal K, Pearson A: Acquired macular disorders; in Kanski J, Bowling B: Clinical Ophthalmology: A Systemic Approach. Saunders Elsevier, ed 7, 2011, pp 629-631.

-5 Krishnan R, Toussounis C, Fung Yang Y: 20-gauge and 23-gauge phacovitrectomy for idiopathic macular holes: comparison of complications and long-term outcomes. Eye 2013;27:72-77.

-6 Takahashi A, Yoshida A, Nagaoka T, et al: Idiopathic full-thickness macular holes and the vitreomacular interface: a high-resolution spectral-domain optical coherence tomography study. Am J Ophthalmol 2012;154:881-892.

7 Uemura A, Otsuji F, Nakano T, Sakamoto T: Vitreomacular interface and outer foveal microstructure in fellow eyes of patients with unilateral macular holes. Retina 2014;34:1229-1234.

$\checkmark 8$ Theodossiadis G, Petrou P, Eleftheriadou M, et al: Focal vitreomacular traction: a prospective study of the evolution to macular hole: the mathematical approach. Eye 2014;28:1452-1460.

9 Gaudric A, Haouchine B, Massin P, et al: Macular hole formation: new data provided by optical coherence tomography. Arch Ophthalmol 1999;117:744-751. 
Case Reports in

Ophthalmology
Case Rep Ophthalmol 2015;6:339-344

DOI: $10.1159 / 000440647$

Ginton et al.: Photoreceptor Outer Segment on Internal Limiting Membrane after

Macular Hole Surgery: Implications for Pathogenesis

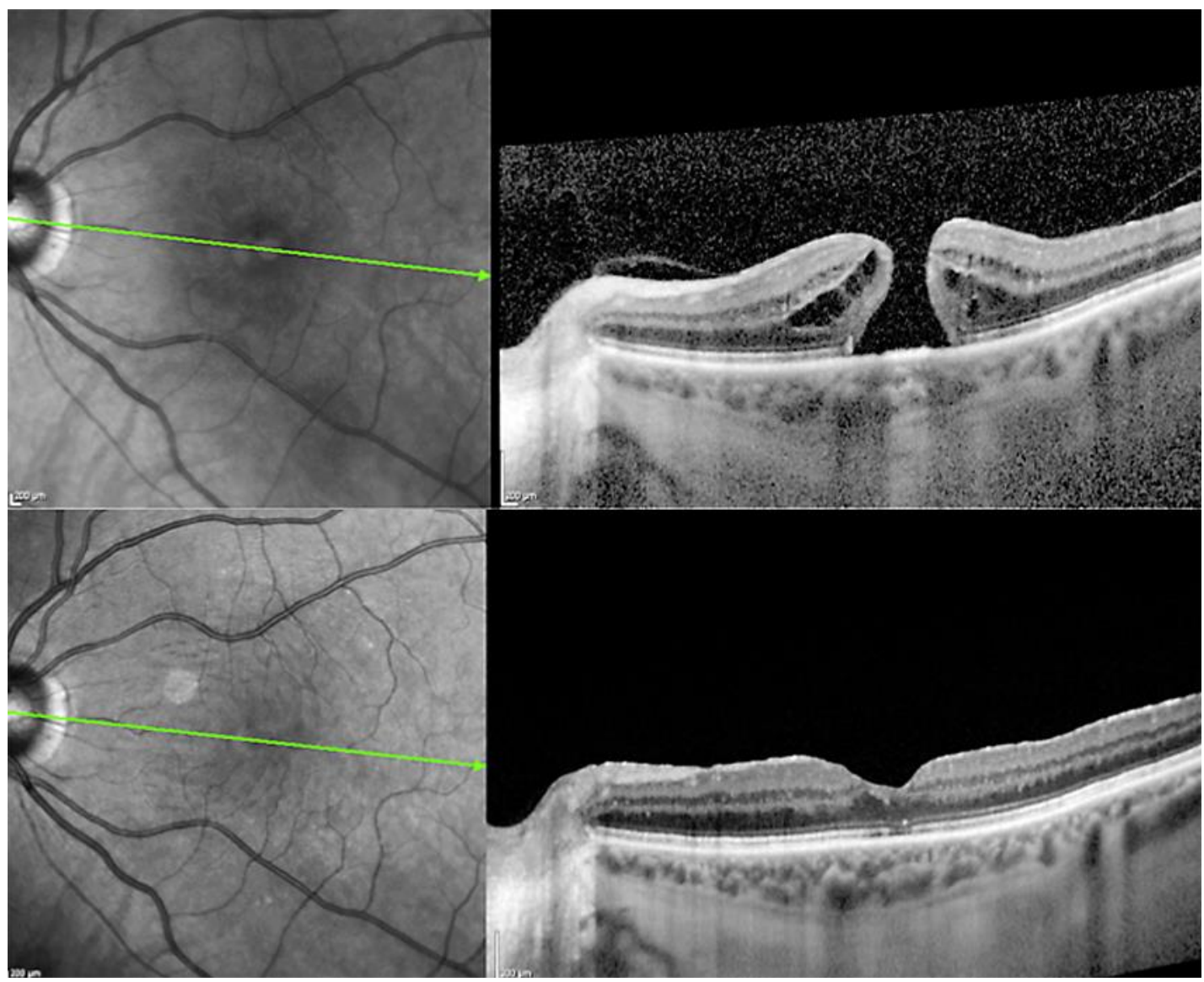

Fig. 1. Pre-operative (above) and post-operative (below) OCT of the left eye. 


\section{Case Reports in \\ Ophthalmology}

\begin{tabular}{l|l}
\hline \multicolumn{2}{l}{ Case Rep Ophthalmol 2015;6:339-344 } \\
\hline DOI: 10.1159/000440647 & $\begin{array}{l}\text { @ 2015 S. Karger AG, Basel } \\
\text { www.karger.com/cop }\end{array}$ \\
\hline
\end{tabular}

Grinton et al.: Photoreceptor Outer Segment on Internal Limiting Membrane after Macular Hole Surgery: Implications for Pathogenesis

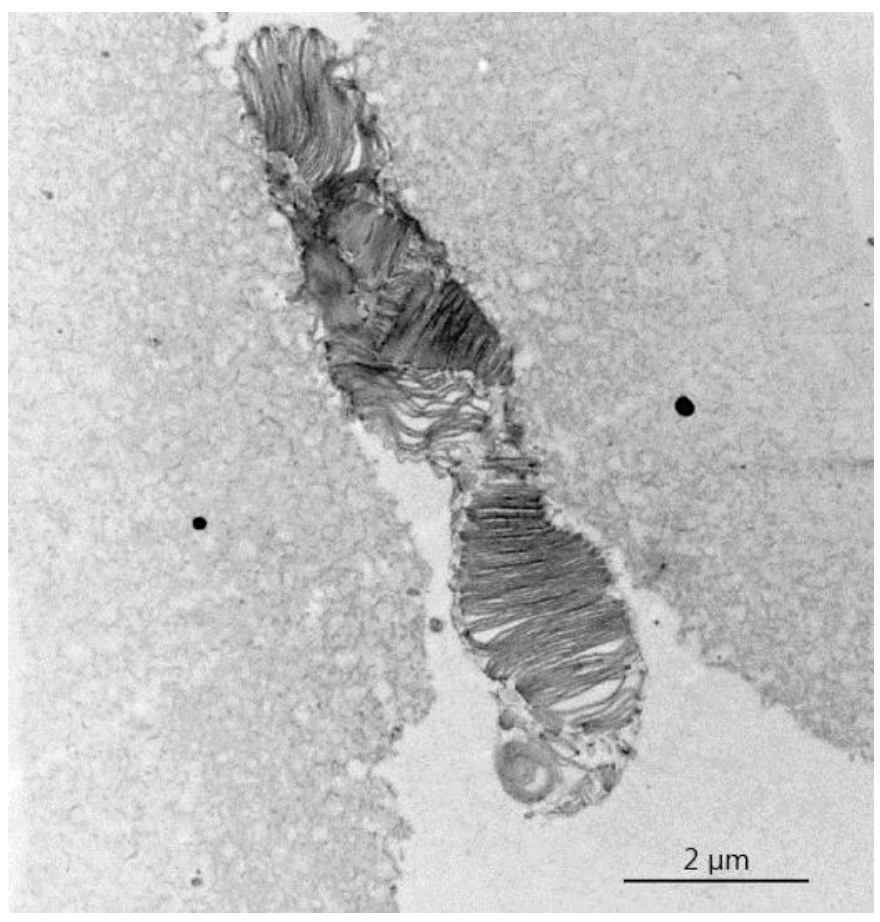

Fig. 2. EM of the left ILM sample, with the photoreceptor outer segment clearly seen. The tissue on either side is folded ILM with the retinal sides abutting the outer segment.

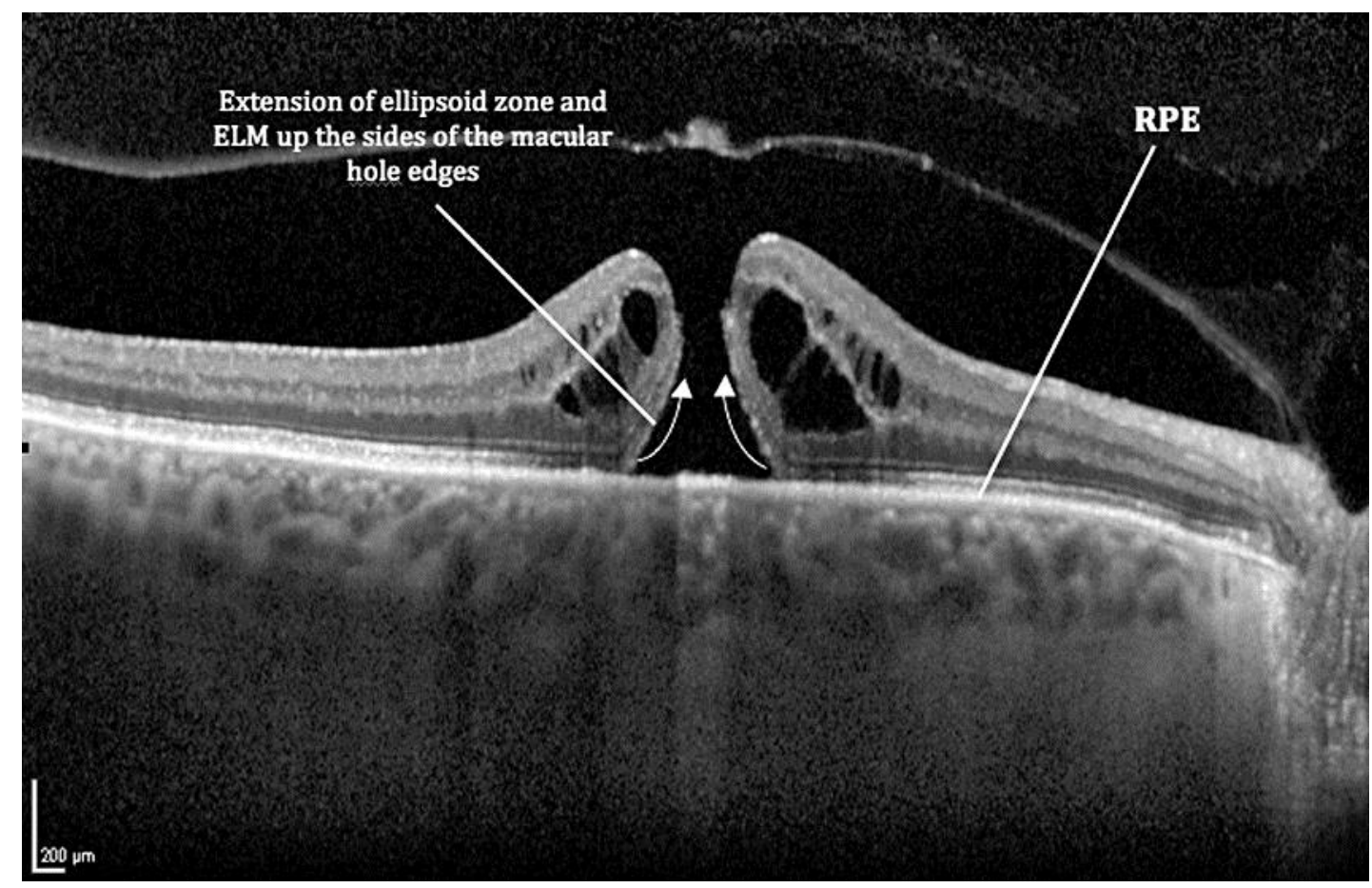

Fig. 3. Extension of the ellipsoid zone and external limiting membrane up the sides of the macular hole edges. 